\title{
Oligomerization and Hemolytic Properties of the C-Terminal Domain of Pyolysin, a Cholesterol Dependent Cytolysin
}

Lisa Pokrajac $^{\mathrm{a}}$, J. Robin Harris ${ }^{\mathrm{bc}}$, Naghmeh Sarraf ${ }^{\mathrm{d}}$ and Michael Palmer ${ }^{\mathrm{ae}}$

a Department of Chemistry, University of Waterloo, 200 University Ave. W., Waterloo, Ontario, N2L 3G1, Canada

${ }^{b}$ Institute for Cell and Molecular Biosciences, University of Newcastle, Newcastle-upon-Tyne, UK 'Institute of Zoology, University of Mainz, 55099 Mainz, Germany).

${ }^{\mathrm{d}}$ Health Sector, Biotechnology Research Institute, National Research Council of Canada, 6100

Royalmount Avenue, Montreal, Quebec, Canada H4P 2R2

${ }^{\mathrm{e} C}$ Corresponding author. Email: mpalmer@uwaterloo.ca 


\begin{abstract}
Pyolysin (PLO) belongs to the homologous family of the cholesterol-dependent cytolysins (CDCs), which bind to cell membranes containing cholesterol to form oligomeric pores of large size. The CDC monomer structure consists of four domains. Among these, the C-terminal domain 4 has been implicated in membrane binding of the monomer, while the subsequent processes of oligomerization and membrane insertion have primarily been assigned to other domains of the molecule. Recombinantly expressed or proteolytic fragments that span the domains 4 of the CDCs streptolysin O (SLO) and perfringolysin O (PFO) bind to membranes but fail to oligomerize, and they inhibit the activity of the respective wild type toxins. We here report that the isolated domain 4 of pyolysin (PLOD4) not only binds to membranes but also forms oligomers with itself, as well as hybrid oligomers with the full-length toxin. As expected, the pure PLO-D4 oligomers are devoid of pore-forming activity. Surprisingly, however, within hybrid oligomers, PLO-D4 not only fails to inhibit, but even amplifies the hemolytic activity of the full-length toxin, to an extent similar to that of doubling the amount of the full-length toxin alone. We propose that this amplification may be related to the kinetics of the oligomerization reaction. Overall, our findings indicate a greater role of domain 4 in the oligomerization of CDCs than previously demonstrated.
\end{abstract}




\section{Introduction}

The cholesterol-dependent cytolysins (CDCs) are a family of homologous pore-forming toxins produced by various species of Gram-positive bacteria (Heuck et al., 2010). Among its members, streptolysin $\mathrm{O}$ (SLO), perfringolysin $\mathrm{O}$ (PFO), pneumolysin and listeriolysin $\mathrm{O}$ have been most thoroughly characterized in terms of structure and function. Crystal structures are available for perfringolysin (Rossjohn et al., 1997) and intermedilysin (Polekhina et al., 2005). In both toxins, and presumably in the other CDCs as well, the tertiary structure is comprised of 4 domains that are arranged into an elongated shape (Figure 1). The C-terminal domain 4 is the only one that is formed by a single contiguous stretch of the primary sequence (Rossjohn et al., 1997). This domain contains the highly conserved tryptophan-rich motif that also, with all CDCs but pyolysin and intermedilysin, contains a unique cysteine residue whose covalent modification prevents membrane binding of the monomeric toxin molecule (Iwamoto et al., 1987). A proteolytic fragment of perfringolysin $\mathrm{O}$ that contained all of domain 4 and some adjoining part of domain 2, the latter most likely in unstructured form, was shown to retain membrane-binding activity, and also to interfere with the hemolytic activity of simultaneously applied intact toxin (Iwamoto et al., 1990; Tweten et al., 1991). The same behaviour was observed with a recombinantly expressed fragment of SLO that contained domain 4 alone (Weis and Palmer, 2001). Therefore, in the overall mode of action of the toxins, domain 4 has been assigned the role of initial membrane binding of the monomer. The subsequent steps of oligomerization and membrane insertion have primarily implicated domain 3 (Palmer et al., 1998; Palmer et al., 1996; Shatursky et al., 1999; Shepard et al., 1998) and domain 1 (Abdel Ghani et al., 1999). While the domain 4 fragment of SLO caused some inhibition of the oligomerization of simultaneously applied full-length SLO, no stable or productive incorporation into hybrid oligomers was observed (Weis and Palmer, 2001). From these findings, it would appear that domain 4 contributes very little to the formation of the membrane-associated CDC oligomer.

Pyolysin, while clearly a member of the CDC toxin family, nevertheless diverges further from the consensus sequence than the other members (Billington et al., 1997). Therefore, we thought it worthwhile to revisit the correlation of structure and function of specific domains with this toxin. In a previous study (Pokrajac et al., 2012), we reported that the effect of an engineered disulfide tether between domains 2 and 3 of the molecule deviates from the effect of the same tether introduced into perfringolysin $\mathrm{O}$ (Hotze et al., 2001), such that it stalls the activity of the toxin already at the level of oligomerization, rather than only at membrane insertion. We here report that the isolated domain 4 
fragment of pyolysin, too, deviates in activity from the aforementioned fragments of streptolysin $\mathrm{O}$ and perfringolysin O. Our present findings show that PLO domain 4 is capable of self-oligomerization, though not of pore formation. The fragment can also form hybrid oligomers with wild type toxin. Surprisingly, the activity of the wild type toxin is not only retained in the presence of the domain 4 fragment but is even amplified by the latter. Hence, compared to other CDC toxins, the isolated domain 4 of PLO retains a decidedly greater scope of function. The findings reported in this study support a greater functional role of domain 4 in the overall activity of CDC than previously reported.

\section{Materials and methods}

Protein expression and purification. The plasmid pJGS59 encoding the PLO gene containing a histidine purification sequence (Billington et al., 1997) was used as the template for PCR to create a cysteinecontaining, functionally intact toxin mutant (N90C) and to express the C-terminal fragment that corresponds to domain 4. The recombinantly expressed C-terminal fragment, PLO-D4, comprises residues 421 to 534 of the native sequence. The $\mathrm{N}$-terminus of the fragment was extended with a hexahistidine tag for purification, a thrombin cleavage site, and a single cysteine residue to facilitate fluorescent labeling. The primers used for this purpose had the following sequences: 5'-CTG GTA CCC AGG GGG TCC TGC TAC AAG TCT GGT GAA ATC ACC-3' (forward), and 5'-GCA GGA CCC CCT GGG TAC CAG ACC ACC ATG ATG ATG ATG ATG ATG AGA ACC-3' (reverse).

PCR products were used to transform the E. coli XL1 Blue strain, cultured on LB ampicillin plates and incubated at $37{ }^{\circ} \mathrm{C}$ for $12-18$ hours. After sequence verification, recombinant plasmids were transformed into E. coli BL21 and cultured in $2 \times$ YT broth supplemented with $0.5 \mathrm{mM}$ IPTG (BioShop, Burlington $\mathrm{ON}$ ) for protein expression. After harvesting, the bacterial cells were lysed using an Emulsiflex C5 Emulsifier (Avestin, Ottawa ON), and the protein was purified using a BioRad Biologic LP liquid chromatography system (Mississauga ON) with nickel agarose column (Qiagen, Mississauga ON). To remove the hexa-histidine purification tag from the PLO-D4 toxin fragment, the protein was equilibrated by gel filtration with thrombin cleavage buffer consisting of $50 \mathrm{mM}$ Tris (BioShop, Burlington $\mathrm{ON}$ ), $150 \mathrm{mM} \mathrm{NaCl}$ (BioShop, Burlington $\mathrm{ON}$ ) and $2.5 \mathrm{mM} \mathrm{CaCl}_{2}, \mathrm{pH}$ 7.5. Human plasma thrombin (Sigma Chemicals, St Louis MO) was added to $1 \mu \mathrm{g}$ for every $100 \mu \mathrm{g}$ of substrate protein, incubated 12-18 hours at $4{ }^{\circ} \mathrm{C}$; the cleavage was monitored using SDS-PAGE. Protein molecular masses were determined on a MicroMass Q-ToF quadropole time of flight mass spectrometer 
(Montreal QC).

Chemical modification of cysteine residues. Protein samples were transferred to labelling buffer consisting of $50 \mathrm{mM}$ Tris, $150 \mathrm{mM} \mathrm{NaCl}, 1 \mathrm{mM}$ EDTA (BioShop, Burlington ON) pH 7.5 using gel filtration. The samples were supplemented with $1 \mathrm{mM}$ of either fluorescein-5-maleimide (Biotium Inc, Hayward CA) or rhodamine red maleimide (Invitrogen, Burlington ON). The samples were incubated at $25^{\circ} \mathrm{C}$ for 60 minutes and the excess label was removed by gel filtration. To determine the labelling efficiency, the molar ratio of fluorophore to protein was calculated from UV-VIS spectra with extinction coefficients of $83,0001 / \mathrm{mol} \times \mathrm{cm}$ for fluorescein at $490 \mathrm{~nm}$ and $91,0001 / \mathrm{mol} \times \mathrm{cm}$ for rhodamine at $540 \mathrm{~nm}$. The extinction coefficient of PLO was determined to be $68,5001 / \mathrm{mol} \times \mathrm{cm}$ at 280 $\mathrm{nm}$. The measured absorbance values at $280 \mathrm{~nm}$ of the labeled proteins were corrected for absorbance of the dyes at $280 \mathrm{~nm}$, using 35,000 1/mol $\times \mathrm{cm}$ and 17,700 1/mol $\times \mathrm{cm}$ for fluorescein and rhodamine, respectively. Labelling efficiencies ranged from $85-90 \%$ for fluorescein and $70-80 \%$ for rhodamine.

Preparation of red blood cells and membrane ghosts. To prepare red blood cells (RBCs), aliquots of $400 \mu \mathrm{l}$ sheep blood were made up to $1 \mathrm{ml}$ with PBS buffer $\left(16 \mathrm{mM} \mathrm{K}_{2} \mathrm{HPO}_{4}, 150 \mathrm{mM} \mathrm{NaCl}, 1 \mathrm{mM}\right.$ EDTA, $\mathrm{pH} 7.5$ ), centrifuged at $380 \times \mathrm{g}$ for 4 minutes, and the supernatant was removed to yield approximately $100 \mu \mathrm{l}$ of RBC pellet. The pellet was washed repeatedly by resuspending with PBS and centrifugation until the supernatant remained clear, and finally resuspended again to a final volume of 1 $\mathrm{ml}$ and a concentration of $10 \%$. From this, 1\% RBC working suspensions were made. To prepare membrane ghosts, $400 \mu \mathrm{l}$ of sheep blood were mixed with $600 \mu \mathrm{l}$ cell lysis buffer $(5 \mathrm{mM} \mathrm{NaCl}, 5 \mathrm{mM}$ $\mathrm{Na}_{2} \mathrm{HPO}_{4}, \mathrm{pH} 7.0$ ) and centrifuged at $16,000 \times \mathrm{g}$ for 10 minutes. The supernatant was removed and the pellet washed repeatedly by centrifugation until both the supernatant became clear and the pellet translucent. The pellet was made up to $1 \mathrm{ml}$ with PBS buffer.

Hemolysis assay. Wild type PLO $(10 \mu \mathrm{l})$ was admixed with PLO-D4 to ratios of 1:1, 1:4 and 1:16. Two-fold serial dilutions were made in a 96-well plate with PBS buffer. Sheep red blood cells were added to each well to a final concentration of $0.5 \%$ and incubated at $37{ }^{\circ} \mathrm{C}$ for 30 minutes. Hemolysis causes a decrease in cell turbidity, which was monitored at a wavelength outside the absorbance of hemoglobin (650 nm) using a SpectroMax Plus 384 microplate spectrophotometer (Molecular Devices, Sunnyvale CA). Hemolytic activity of the wild type PLO/D4 mixtures was compared to wild type PLO alone and PLO-D4 alone. The same samples were tested for lysis kinetically at $25^{\circ} \mathrm{C}$ for 30 minutes.

Fluorescence measurement and data analysis. All samples containing labelled protein were made to a 
final concentration of $1 \mu \mathrm{M}$ with $1 \%(\mathrm{v} / \mathrm{v})$ of red cell membrane ghosts. For hybrid oligomer analyses, the samples containing fluorescein-labelled PLO-N90C (PLO-N90C-F) and rhodamine-labelled PLON90C (PLON90C-R) were mixed to a 1:2 ratio (where PLO-N90C-F concentration was $0.33 \mu \mathrm{M}$ and PLO-N90C-R concentration was $0.66 \mu \mathrm{M}$ ). The hybrid samples containing rhodamine-labelled PLOD4 (PLO-D4-R) and fluorescein-labelled (PLO-N90C-F) were also mixed to a 1:2 ratio (where PLOD4-R concentration was $0.33 \mu \mathrm{M}$ and PLO-N90CF-F concentration was $0.66 \mu \mathrm{M}$ ).

For all other fluorescence assays, fluorescein- and rhodamine-labelled toxin was combined in a 1:1 ratio $\left(0.5 \mu \mathrm{M}\right.$ each). After incubation at $37^{\circ} \mathrm{C}$ for 30 minutes, the protein-membrane samples were centrifuged at $16,000 \times \mathrm{g}$ for 10 minutes, the supernatant removed, and the pellet resuspended with PBS. Steady state fluorescence spectra were recorded for soluble toxin controls, membrane-bound samples, and the centrifugation supernatant. The intensity of the membrane-bound samples was corrected for incomplete binding toxin according to the following equation:

$$
F_{\text {membrane, corrected }}=\frac{F_{\text {membrane, raw }}}{F_{\text {solution }}-F_{\text {supernatant }}}
$$

where $F_{\text {solution }}$ is the intensity of the toxin prior to incubation with membranes, and $F_{\text {supernatant }}$ is the intensity of the supernatant after incubation with membranes and centrifugation. All sample spectra were subtracted with buffer or erythrocyte ghost suspension blanks as appropriate.

Steady state fluorescence measurements were conducted on a PTI QuantaMaster spectrofluorimeter using an excitation wavelength of $465 \mathrm{~nm}$ with emission scans recorded from 485 to $630 \mathrm{~nm}$, using 2 $\mathrm{nm}$ band passes. Time-resolved fluorescence was measured using an FT-100 compact fluorescence lifetime spectrometer (PicoQuant, Berlin, Germany) using an LDH-PC-470 LED laser light source. Fluorescein emission was isolated using a $520 \pm 5 \mathrm{~nm}$ bandpass filter (Andover Corporation, New Hampshire). Decays were fitted using FluoFit software (PicoQuant) using three exponential lifetime components, from which the average lifetime $<\tau>$ was calculated according to

$$
\tau \geq \frac{\sum \alpha_{i} \tau_{i}}{\sum \alpha_{i}}
$$

where $\alpha_{i}$ represents the amplitude at time zero and $\tau_{i}$ is the lifetime of the $i^{\text {th }}$ component. The threeexponential fits yielded $\chi^{2}$ values $\leq 1.2$. 
Oligomer size characterization by size exclusion chromatography. In order to compare the relative sizes of hybrid oligomer complexes, samples of labelled PLO-N90C-F or PLO-D4-F were made to 1 $\mu \mathrm{M}$ concentration, with varying concentrations of unlabelled PLO-D4 added as indicated in the Results section. Membrane ghosts were added to a final concentration of $20 \%(v / v)$. Samples were incubated at $37{ }^{\circ} \mathrm{C}$ for 30 minutes, centrifuged at $16,000 \times \mathrm{g}$ for 10 minutes, after which the supernatant was removed. Membranes were dissolved using 5\% sodium deoxycholate, and the samples were brought to a final volume of $1 \mathrm{ml}$. Size exclusion chromatography was performed on a BioRad BioLogic chromatography system using a Sephacryl S-400 column equilibrated with elution buffer consisting of $20 \mathrm{mM}$ Tris, $150 \mathrm{mM} \mathrm{NaCl}, 1 \mathrm{mM}$ EDTA and 0.25\% (w/v) sodium deoxycholate (BioShop, Burlington $\mathrm{ON}) \mathrm{pH}$ 8.5. Eluted fractions were collected and analyzed for fluorescein fluorescence.

Transmission electron microscopy. Wild type PLO and the C-terminal fragment PLO-D4, alone or in mixtures as indicated in the Results section, were incubated at a total protein concentration of 0.125 $\mathrm{mg} / \mathrm{ml}$ with cholesterol crystals $0.5 \mathrm{mg} / \mathrm{ml}$, prepared according to published procedures (Harris et al., 1998 ) for 30 minutes at room temperature. The samples were subjected to negative staining with $2 \%$ uranyl acetate. Transmission electron microscopy study of the negatively stained specimens was performed by a Phillips CM 100 transmission electron microscope at $100 \mathrm{kV}$. Digital images were recorded using an Optronics $1824 \times 1824$ pixel CCD camera with an AMT40 version 5.42 capture engine supplied by Deben (Bury St. Edmunds, UK). All TEM analyses were performed at the University of Newcastle, Newcastle upon Tyne, UK.

\section{Results}

Structural integrity of PLO-D4. A CD spectrum of PLO-D4 was used to estimate the secondary structure using the K2D3 web service (http://www.ogic.ca/projects/k2d3/) (Louis-Jeune et al., 2011). The predicted structure $-38 \%$ beta structure and $<2 \%$ alpha helix, with the remainder being random coil - suggests that the folded structure of the fragment resembles that in the intact protein (compare Figure 1). This is also supported by the scope of residual function of the fragment, which exceeds that of previously characterized homologous toxin fragments (see below).

Hemolytic activity of PLO-D4 alone and in combination with wild type PLO. In order to test the activity of PLO-D4, the protein was combined with sheep erythrocytes, and the extent of hemolysis was determined by the decrease in turbidity $\left(\mathrm{OD}_{650}\right)$ of the cell suspension (Figure 2). In this assay, 
wild type PLO (PLO-WT) achieved 50\% hemolysis at approximately $0.4 \mu \mathrm{g} / \mathrm{ml}$, which is similar to previous measurements (Ikegami et al., 2000). As expected from previous studies on perfringolysin $\mathrm{O}$ and streptolysin O (Tweten et al., 1991; Weis and Palmer, 2001), PLO-D4 alone shows no hemolytic activity. However, when the fragment is combined with PLO-WT, the hemolytic activity of the mixture exceeds that of PLO-WT alone.

Under the experimental conditions of Figure 2, the enhancement of the hemolytic activity of PLO-WT by PLO-D4 is manifest only at one concentration of the wild type toxin $(0.312 \mu \mathrm{g} / \mathrm{ml})$, while at 0.625 $\mu \mathrm{g} / \mathrm{ml}$ virtually the same end point is reached with and without the domain 4 fragment.

The enhancement of the hemolytic activity of PLO-WT by PLO-D4 was consistently observed in several repeated experiments, and in each single experiment its extent was very regularly correlated with the amount of PLO-D4 present. Between experiments, however, the absolute extent of hemolysis caused by given amounts of PLO-WT and PLO-WT was somewhat variable, such that the standard deviations of the data points representing different concentrations of PLO-D4 in Figure 2 would overlap each other (data not shown).

The enhancement of hemolysis by the PLO-D4 fragment can also be observed as a dose-dependent acceleration of hemolysis in a kinetic assay (Figure 3A). Interestingly, the extent of this acceleration is limited and appears to level off with increasing excess of the fragment over wild type PLO.

For comparison, the time course of hemolysis observed with various amounts of PLO-WT is shown in Figure 3B. The effect of a single additional equivalent of PLO-WT on the rate of hemolysis appears similar to that of 16 equivalents of PLO-D4, and higher concentrations of wild type toxin increase the speed of hemolysis even further. The observation that the promotion of hemolysis by domain 4 can be saturated suggests that its productive interaction with PLO-D4 is limited by the amount of the latter. Nevertheless, the finding that PLO-D4 increases the hemolytic activity of wild type PLO is in stark contrast with previous observations on the isolated domain 4 of streptolysin O (Weis and Palmer, 2001) as well as with a similar proteolytic fragment of perfringolysin O (Iwamoto et al., 1990), both of which inhibit rather than augment the activities of the respective wild type toxins.

Oligomerization of PLO-D4. To examine the ability of PLO-D4 to form oligomers on its own or with the full length toxin, both molecules were thiol-specifically labelled with fluorescein and rhodamine, respectively. The two labels form a donor-acceptor pair for fluorescence energy transfer (FRET) studies with a Förster distance $\left(\mathrm{R}_{0}\right)$ of approximately $50 \AA$ (Wu and Brand, 1994). The fluorescein-labelled 
(PLO-D4-F) and the rhodamine-labelled fragment (PLO-D4-R) were mixed and then incubated with sheep erythrocyte membrane ghosts, and the fluorescence emission was measured (Figure 4A). The pronounced decrease in the donor (fluorescein) signal when combined with acceptor (rhodamine) indicates that the two PLO-D4 fragments can associate with each other and thereby engage in FRET. When the two species were applied to the membranes sequentially rather than simultaneously, the donor signal was weaker than with PLO-D4-F alone but stronger than with the premixed sample. This suggests that at least some of the molecules in the initially applied sample were sequestered in oligomers and no longer available for the formation of hybrid oligomers at the time of the second application. This result is consistent with the formation of oligomers that are stable on the time scale of the experiment. The attenuation of donor fluorescence in the combined samples is not the result of competition for binding sites by the acceptor-labelled fragments, as application of two equivalents of donor also doubles the donor intensity (data not shown).

Oligomers of SLO and PFO are stable after membrane disruption with detergents, and solubilization with deoxycholate has been used to isolate and characterize these oligomers (Palmer et al., 1996). In order to test the stability of the domain 4 oligomers towards dissociation by detergents, PLO-D4-R and PLO-D4-F were incubated with membrane ghosts and subsequently with deoxycholate to dissolve the membranes. Fluorescence spectra were obtained before and after deoxycholate solubilization (Figure 3B). The intensity of the fluorescein peak increases greatly upon membrane solubilization, indicating disruption of the oligomers and abolition of FRET. In contrast, the oligomers of an active, labeled fulllength toxin mutant (N90C) readily withstand membrane solubilization, as evident from the continued existence of FRET before and after solubilization. This indicates that oligomers formed by domain 4 alone are linked by forces weaker than those occurring in oligomers of intact toxin molecules.

It may also be noted that, in Figure 4B, the rhodamine fluorescence emission is stronger with the full length toxin than with the domain 4 fragment. Rhodamine is subject to concentration-dependent selfquenching (MacDonald, 1990), and it appears possible that this effect is stronger with the domain 4 fragment than with the full length toxin. This effect does not change the interpretation of the experiment.

Morphology of PLO-D4 oligomers. Cholesterol has been shown to induce oligomerization of streptolysin O (Duncan and Schlegel, 1975), and cholesterol crystals have been established as a useful model system to study the oligomerization of CDCs by EM (Harris et al., 1998; Harris et al., 2011). On 
such crystals, intact wild type PLO forms the usual ring and arc structures (Figure 5A) that have also been observed with many other CDCs. In contrast, PLO-D4 forms a pattern of parallel lines (Figure 5B). Evidently, the absence of the first three domains profoundly changes the size and shape of the oligomers formed by PLO-D4, but does not abrogate oligomer formation as such.

Formation of hybrid oligomers by PLO-D4 and wild type PLO. From the observation of oligomers of both PLO-D4 and of the full-length molecule, the question arises if the two can also form hybrid oligomers. The enhancement of the hemolytic activity of wild type PLO by PLO-D4 suggests that this should possible. In order to directly detect such hybrids, PLO-D4-R was mixed with PLO-N90C-F, and the mixture was incubated with membranes. Formation of hybrid oligomers should result in FRET between the two species, and this is indeed apparent from the reduction in PLO-N90C-F fluorescein emission (Figure 6A). FRET is pronounced only when the two species are applied simultaneously, but only minor upon sequential application, which corroborates the conclusion that is is mostly due to the formation of hybrids, as opposed to FRET between segregated donor- and acceptor-labelled oligomers that happen to be located in close proximity to each other.

In hybrid oligomers, the domain 4 fragment might be restricted to terminal positions, or alternatively it might intercalate between two molecules of full-length toxin. In order to determine whether or not such intercalation occurs, fluorescence measurements were performed on an equimolar mixture of PLON90C-F and PLO-N90C-R, to which increasing amounts of unlabelled PLO-D4 were added. The rationale here is that intercalation of PLO-D4 between the donor- and acceptor-labelled full-length molecules should reduce FRET between the latter. Figure 6B shows that, with increasing PLO-D4 concentration, the donor signal also increases, and the acceptor signal decreases. This change is small but reproducible, and is indicative of a slight increase in the average spacing between the labelled fulllength toxin monomers. However, as also shown in Figure 6B, the decrease in energy transfer was far more pronounced when unlabelled wild type PLO was employed instead of the unlabelled D4 fragment.

These observations are corroborated by time-resolved fluorescence measurements (Table 1). Fluorescein-labelled N90C alone yields a fluorescein lifetime of $2.8 \mathrm{~ns}$, whereas the mixture of N90C-F and N90C-R without D4 yields a lifetime of 1.1 ns. When the unlabelled D4 fragment is added, the average lifetime of fluorescein increases slightly, while a much greater increase is seen with unlabelled wild type PLO. An increase in the lifetime of the donor reflects an increase in quantum yield, and thus 
a decrease in the efficiency of FRET. Therefore, the collective results indicate that the domain 4 fragments can intercalate between full length toxin molecules, but with an efficiency that is very greatly reduced relative to the intact toxin molecules themselves.

Conceivably, the low efficiency of PLO-D4 intercalation between subunits of full-length toxin might be explained by substantially different rates of oligomerization. If one species undergoes oligomerization much more rapidly than the other, this would favour the formation of segregated oligomers. To compare the rates of oligomerization, the time course of fluorescein fluorescence was monitored during incubation with red cell membrane ghosts of a mixture of PLO-N90C-F and PLO-N90C-R, as well as of a mixture of PLO-D4-F and PLO-D4-R, and also of a mixture PLO-N90C-F and PLO-D4-R (Figure 7). Progress of oligomerization is evident from the decrease in fluorescein fluorescence due to FRET. While the kinetic curves of the different samples vary somewhat in shape and slope, overall the rates of change in donor fluorescence are of comparable magnitude. This suggests that differences in the rate of oligomerization are not the major reason for the relatively low efficiency of intercalation of domain 4 between full-length toxin molecules in mixed samples.

Morphology of hybrid oligomers. On crystalline cholesterol surfaces, mixtures of PLO-WT and PLOD4 yield rings and arcs as expected for PLO-WT alone, as well as linear stripes as expected for PLOD4 alone (Harris et al., 2011). However, occasionally, the two shapes are seen to combine into walkingcane like formations (Figure 5C and D). It appears likely that the arc portion of the walking cane oligomer is formed predominantly by wild type molecules, whereas the stick consists mostly of domain 4 fragment. The observed structures appear compatible with the notion of a limited but measurable ability of domain 4 to form hybrid oligomers with PLO-WT, as inferred from fluorescence experiments.

\section{Discussion}

The findings in this study clearly demonstrate that the isolated domain 4 fragment of PLO differs in several ways from similar fragments of PFO and SLO (Iwamoto et al., 1990; Tweten et al., 1991; Weis and Palmer, 2001). Unlike those previously characterized fragments, PLO-D4 forms homogeneous oligomers on membranes, can form hetero-oligomers with the wild type toxin, and amplifies rather than inhibits the hemolytic activity of the latter. These observations raise several intriguing questions.

One question concerns the mechanism of the amplification of the hemolytic activity of the full-length toxin. It has been clearly established that domain 3 contains the crucial segments of the toxin molecule 
that insert into the membrane (Palmer et al., 1996) to form the trans-membrane $\beta$-barrel (Shatursky et al., 1999; Shepard et al., 1998), while domain 4 resides on the outer surface of the target membrane even in the fully inserted, final form of the oligomer and does not directly contribute to the membranespanning parts of the pore (Tilley et al., 2005). In keeping with this model, the PLO domain 4 fragment itself, like the corresponding SLO and PFO fragments, is devoid of any hemolytic activity. Therefore, the observed amplification most likely comes about in a more indirect manner.

One possible indirect mechanism relates to the kinetics of oligomerization. With SLO, the oligomerization reaction has been found to follow a two-step, nucleation-extension mechanism (Palmer et al., 1995). In this mechanism, the nucleation step is of second order for the toxin monomer and is rate-limiting for the overall reaction. In limiting dilution hemolysis experiments, hemolysis likely ceases because the nucleation step no longer proceeds efficiently on the time scale of the experiment. Assuming that the nucleation reaction normally involves two molecules of wild type toxin, it appears possible that the domain 4 fragment may substitute one or both of these two molecules, thus accelerating the nucleation reaction.

Interestingly, the extent of hemolytic amplification becomes self-limiting at very high concentrations of PLO-D4. One possible explanation is that, as depicted in Figure 8C, one of the partners in the nucleation reaction has to be a wild-type molecule, the concentration of which therefore becomes ratelimiting for nucleation at large excess of PLO-D4. Another possibility is that, while nuclei formed from two molecules of PLO-D4 can recruit wild-type monomers (Figure 8D), this process is limited by the competing mutual aggregation of PLO-D4 nuclei and monomers. Either way, it is interesting to note that hemolytic activity levels off but does not decrease at very large excess of PLO-D4. This suggests that PLO-D4 interacts assists in priming the oligomerization of wild type toxin but does not interfere with its further progress. This hypothetical scenario seems to agree with the experimental evidence suggesting that even in hybrid oligomers the full-length and the domain 4 subunits remain largely segregated. According to fluorescence experiments, intercalation of PLO-D4 between full-length molecules is minimal; most full-length molecules will therefore be able to productively interact with other full-length molecules in adjacent positions.

According to the fluorescence assay shown in Figure 7, the segregation of PLO-WT and PLO-D4 is not accounted for by large differences in the respective rates of oligomerization. Another possible explanation might be that full-length molecules are able to displace domain 4 molecules from the 
growing end of a growing oligomer. The fact that domain 4 oligomers but not full-length oligomers can be disrupted by deoxycholate solubilization (Figure 4B) suggests that the fragment molecules associate more weakly, which might be conducive to such displacement.

The very limited extent of intercalation of domain 4 fragments between full-length molecules inferred from fluorescence experiments agrees with the appearance of putative hybrids in electron microscopy (Figure 5). These walking cane-like structures combine linear segments, presumably containing predominantly PLO-D4 molecules, with curved ones, which likely contain mostly the full-length toxin. It has previously been shown that incomplete, arc-shaped oligomers of SLO and PLO can form functional pores; therefore, it appears likely that the "handles" of the walking canes are also capable of membrane permeabilization.

Regarding the walking cane structures, a possible objection may be that the abutting linear and curved segments are not really connected, but are in fact separate PLO-D4 and full-length toxin oligomers whose ends only came to be in the same place by chance. Note, however, that in each of the instances shown in Figures 5C and D, the direction of the straight segment is smoothly continued by the tangent of the curved section at the meeting point. If there were no intermolecular forces between them, the two structures should be seen meeting at various angles, and a kink going one way or the other should usually be visible between them. Since this is not the case, we propose that the walking cane structures represent true hybrid oligomers.

In summary, our results show that the C-terminal domain of PLO retains the ability to bind to target membranes, to form oligomers with itself, and to form hybrid oligomers with intact toxin molecules to the point of enhancing their activity. The formation of oligomers, as well as the amplification of the activity of the full-length toxin distinguish PLO-D4 from similar fragments of SLO and PFO. Our study provides an intriguing example of how the same experimental strategy for assigning structure and function may lead to different results with homologous protein molecules, and it supports a greater functional role of domain 4 in CDC pore formation than has previously been documented.

Acknowledgements: We thank Martha Su Ma for help with CD spectroscopy, and two anonymous reviewers for useful comments and suggestions. This study was supported by an operating grant from NSERC to M. Palmer. 


\section{References}

Abdel Ghani, EM., Weis, S., Walev, I. et al. (1999). Streptolysin O: inhibition of the conformational change during membrane binding of the monomer prevents oligomerization and pore formation. Biochemistry $38,15204-15211$.

Billington, SJ., Jost, BH., Cuevas, WA. et al. (1997). The Arcanobacterium (Actinomyces) pyogenes hemolysin, pyolysin, is a novel member of the thiol-activated cytolysin family. J Bacteriol 179, 6100-6106.

Duncan, JL. and Schlegel, R. (1975). Effect of streptolysin O on erythrocyte membranes, liposomes, and lipid dispersions. A protein-cholesterol interaction. J Cell Biol 67, 160-174.

Harris, JR., Adrian, M., Bhakdi, S. et al. (1998). Cholesterol-Streptolysin O Interaction: An EM Study of Wild-Type and Mutant Streptolysin O. J Struct Biol 121, 343-355.

Harris, JR., Lewis, RJ., Baik, C. et al. (2011). Cholesterol microcrystals and cochleate cylinders: attachment of pyolysin oligomers and domain 4. J Struct Biol 173, 38-45.

Heuck, A., Moe, P. and Johnson, B. (2010). The Cholesterol-Dependent Cytolysin Family of GramPositive Bacterial Toxins. Subcell Biochem 51, 551-577.

Hotze, E., Wilson-Kubalek, E., Rossjohn, J. et al. (2001). Arresting pore formation of a cholesteroldependent cytolysin by disulphide trapping synchonizes the insertion of the transmembrane betasheet from a pre-pore intermediate. J Biol Chem 276, 8261-8268.

Ikegami, M., Hashimoto, N., Kaidoh, T. et al. (2000). Genetic and biochemical properties of a hemolysin (pyolysin) produced by a swine isolate of Arcanobacterium (Actinomyces) pyogenes. Microbiol Immunol 44, 1-7.

Iwamoto, M., Ohno-Iwashita, Y. and Ando, S. (1987). Role of the essential thiol group in the thiolactivated cytolysin from Clostridium perfringens. Eur J Biochem 167, 425-430.

Iwamoto, M., Ohno-Iwashita, Y. and Ando, S. (1990). Effect of isolated C-terminal fragment of thetatoxin (perfringolysin O) on toxin assembly and membrane lysis. Eur J Biochem 194, 25-31.

Louis-Jeune, C., Andrade-Navarro, MA. and Perez-Iratxeta, C. (2011). Prediction of protein secondary structure from circular dichroism using theoretically derived spectra. Proteins, . 
MacDonald, RI. (1990). Characteristics of self-quenching of the fluorescence of lipid-conjugated rhodamine in membranes. J Biol Chem 265, 13533-13539.

Palmer, M., Harris, R., Freytag, C. et al. (1998). Assembly mechanism of the oligomeric streptolysin O pore: the early membrane lesion is lined by a free edge of the lipid membrane and is extended gradually during oligomerization. EMBO J 17, 1598-1605.

Palmer, M., Saweljew, P., Vulicevic, I. et al. (1996). Membrane-penetrating domain of streptolysin O identified by cysteine scanning mutagenesis. J Biol Chem 271, 26664-26667.

Palmer, M., Valeva, A., Kehoe, M. et al. (1995). Kinetics of streptolysin O self-assembly. Eur J Biochem 231, 388-395.

Pokrajac, L., Baik, C., Harris, R. et al. (2012). Partial oligomerization of pyolysin induced by disulfidetethered mutant. Biochem Cell Biol in press, .

Polekhina, G., Giddings, KS., Tweten, RK. et al. (2005). Insights into the action of the superfamily of cholesterol-dependent cytolysins from studies of intermedilysin. Proc Natl Acad Sci U S A 102, 600-605.

Rossjohn, J., Feil, S., McKinstry, W. et al. (1997). Structure of a cholesterol-binding, thiol-activated cytolysin and a model of its membrane form. Cell 89, 685-692.

Shatursky, O., Heuck, A., Shepard, L. et al. (1999). The mechanism of membrane insertion for a cholesterol-dependent cytolysin: A novel paradigm for pore-forming toxins. Cell 99, 293-299.

Shepard, L., Hueck, A., Hamman, B. et al. (1998). Perfringolysin O: an alpha-helical to beta-sheet transition identified by fluorescence spectroscopy. Biochemistry 37, 293-299.

Tilley, SJ., Orlova, EV., Gilbert, RJC. et al. (2005). Structural basis of pore formation by the bacterial toxin pneumolysin. Cell 121, 247-256.

Tweten, RK., Harris, RW. and Sims, PJ. (1991). Isolation of a tryptic fragment from Clostridium perfringens theta-toxin that contains sites for membrane binding and self-aggregation. J Biol Chem 266, 12449-12454. 
Weis, S. and Palmer, M. (2001). Streptolysin O: the C-terminal, tryptophan-rich domain carries functional sites for both membrane binding and self-interaction but not for stable oligomerization. Biochim Biophys Acta 1510, 292-299.

Wu, P. and Brand, L. (1994). Resonance energy transfer: methods and applications. Anal Biochem 218, $1-13$. 


\section{Table 1}

N90C-fluorescein fluorescence lifetimes in hybrid oligomers. An equimolar mixture of the fluoresceinlabelled and rhodamine-labelled forms of mutant N90C was incubated with membranes, either alone (leftmost column) or with the D4 fragment or wild type PLO added at different molar excess over the labelled N90C. The fluorescein time-dependent fluorescence decay was fit with a three-exponential model, and the average lifetimes $\langle\tau>$ calculated as detailed in the Methods section. The fluoresence lifetime of N90C-F alone after incubation with membranes is shown for comparison. The $\chi^{2}$ values are those of the three-exponential fit.

\begin{tabular}{cllllllll} 
& N90C-F/R & D4 & \multicolumn{3}{c}{ wt } & \multicolumn{2}{l}{ N90C-F } \\
$\begin{array}{c}\text { Unlabeled protein (mol/mol) } \\
<\tau>(\mathrm{ns})\end{array}$ & - & 1 & 2 & 4 & 1 & 2 & 4 & - \\
$\chi^{2}$ & 1.10 & 1.23 & 1.25 & 1.29 & 1.87 & 2.23 & 2.14 & 2.77 \\
& 1.15 & 1.12 & 1.07 & 1.15 & 1.12 & 1.06 & 1.13 & 1.03
\end{tabular}




\section{Legends to Figures}

Figure 1: Structural model of pyolysin, based on the crystal structure of the homologous toxin perfringolysin O (Rossjohn et al., 1997).

Figure 2: Hemolytic activity of wild type PLO with increasing ratios of PLO-D4. The extent of hemolysis of $0.5 \%$ sheep red blood cells after incubation at $37{ }^{\circ} \mathrm{C}$ for 30 minutes was determined by the decrease in turbidity $\left(\mathrm{OD}_{650}\right)$. The concentration of PLO-WT was varied by serial twofold dilution from $2.5 \mu \mathrm{g} / \mathrm{ml}$; the concentration of PLO-D4 in the mixed samples is implied by the molar ratios indicated on the graph (PLO-WT:PLO-D4). The sample containing PLO-D4 only had a concentration of $5 \mu \mathrm{g} / \mathrm{ml}$. The figure shows a single representative experiment.

Figure 3: Time Course of hemolysis of wild type PLO with increasing ratios of PLO-D4. In (A), wild type PLO at $0.625 \mu \mathrm{g} / \mathrm{ml}$ was incubated with $0.5 \% \mathrm{RBC}$ 's at room temperature, and the progress of hemolysis was monitored by the decrease in turbidity $\left(\mathrm{OD}_{650}\right)$. The toxin was present alone $(1: 0)$ or with PLO-D4 at various molar proportions as indicated. The sample labelled 0:1 contains PLO-D4 only. In (B), wild type toxin alone was used at various multiples of the initial concentration (1:0 again equals $0.625 \mu \mathrm{g} / \mathrm{ml})$.

Figure 4: Characterization of domain 4 fragment oligomers by FRET.

A: PLO-D4 labelled with fluorescein (D4-F) alone or in combination with rhodamine (D4-R) were incubated with sheep erythrocyte ghosts. Addition of D4-R prior to incubation with membranes greatly reduces the fluorescence of D4-F due to FRET, indicating oligomerization. If PLO-D4-R is added after incubation for 30 minutes with D4-F alone, the extent of FRET is reduced, indicating that the two species remain partially segregated.

B: A mixture of fluorescein- and rhodamine-labelled PLO-D4 was incubated with membranes as before and the sample solubilized with deoxycholate (DOC). FRET is reversed, indicating dissociation of the oligomer. The same treatment does not change the fluorescence of a sample of N90C-F and N90C-R. 
Figure 5: Electron microscopy of wild type PLO and domain 4 oligomers on cholesterol crystals.

A: Wild type PLO alone. The rings and arcs resemble those previously described for other CDCs.

B: PLO-D4 alone. Instead of rings and arcs, straight rods in parallel arrangement are seen.

C and D: Mixtures of wild type with PLO-D4 in twofold or fivefold excess, respectively. In addition to the arcs, the rings and rods are also observed in A and B, 'walking canes' and other hybrid shapes are seen, some of which are marked with arrows. Black scale bars correspond to $100 \mathrm{~nm}$.

Figure 6: Formation of hybrid oligomers from wild-type PLO and the domain 4 fragment (FRET).

A: PLO-N90C labelled with fluorescein (PLO-N90C-F) and rhodamine (PLO-N90C-R) were incubated with sheep erythrocyte ghosts. FRET (as detected by a decrease of fluorescein fluorescence) only occurs if the two proteins are applied simultaneously. The absence of FRET observed with sequential application, with incubation for 30 minutes between the two species, likely indicates the formation of segregated oligomers.

B: FRET between N90C-F and the rhodamine-labelled species (N90C-R) is effectively suppressed if the labelled toxin is admixed with an equivalent amount of unlabelled wild type toxin before addition to membranes. In contrast, only a very slight reduction of FRET is observed even with a fourfold excess of unlabelled D4 fragment. This indicates that the fragment does not efficiently intercalate between N90C subunits in hybrid oligomers.

Figure 7: Kinetics of oligomer formation on membrane ghosts by FRET. The progress of oligomerization of full-length PLO, the domain 4 fragment and of hybrid oligomer formation was monitored by the decrease of fluorescein fluorescence caused by FRET from fluorescein to rhodamine. Fluorescein- and rhodamine-labelled N90C or D4 fragment were mixed as indicated. At $\mathrm{t}=0$, RBC membrane ghosts were added to $1 \%$, and the sample mixed by pipetting. The spikes at $\mathrm{t}=0$ are artifacts caused by opening and closing the sample compartment. 
Figure 8: Oligomerization of PLO-WT and PLO-D4, and hypothetical mechanism of hemolysis enhancement by PLO-D4 through hybrid oligomer formation.

A: Wild type pyolysin forms arc-shaped (as shown) and ultimately ring-shaped oligomers. Based on studies on the homologous toxin streptolysin O (Palmer et al., 1995), it is likely that the initial reaction between two monomers is the rate-limiting step in oligomerization.

B: The domain 4 fragment alone forms oligomers with linear shape, possibly influenced by the molecular arrangement of the underlying cholesterol substrate (see Figure 5B).

C: In a mixture of wild-type PLO and PLO-D4, the latter might replace one of the wild-type toxin molecules in the rate-limiting nucleation reaction, and thereby enhance the hemolytic activity of wildtype PLO. Addition of both wild-type and D4 fragment molecules to the hybrid nucleus could result in walking-cane structures as those seen in Figures 5C and 5D.

D: In an alternative scenario, a nucleus formed from PLO-D4 alone might react with a wild-type molecule; the ensuing hybrid would again form a walking cane by recruit additional wild type and D4 molecules. 
Figure 1

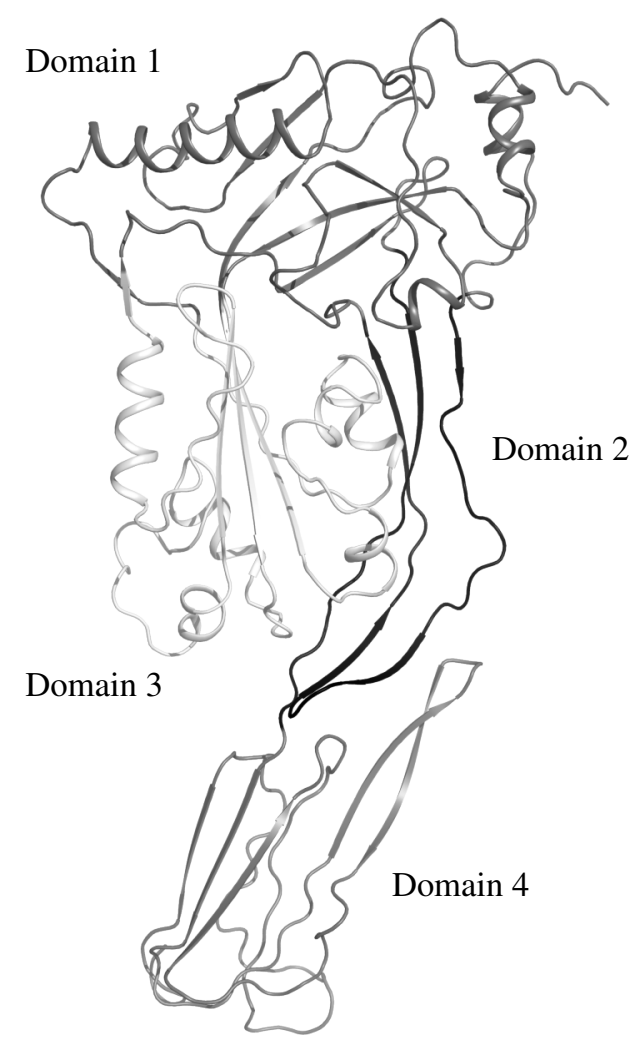


Figure 2

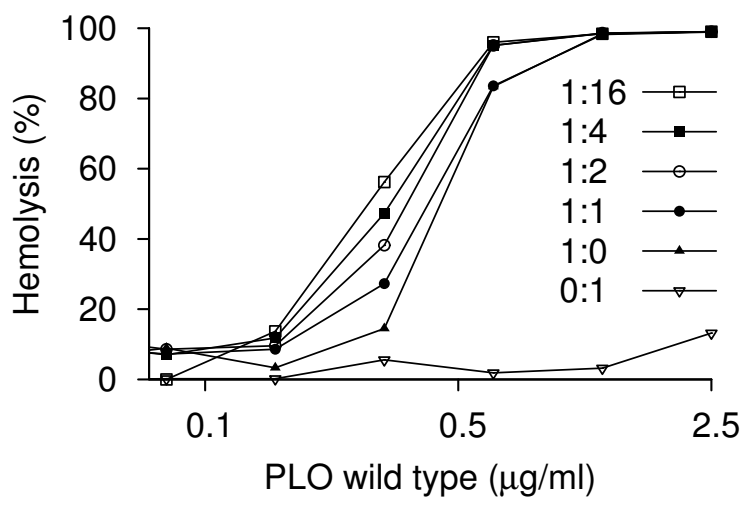


Figure 3
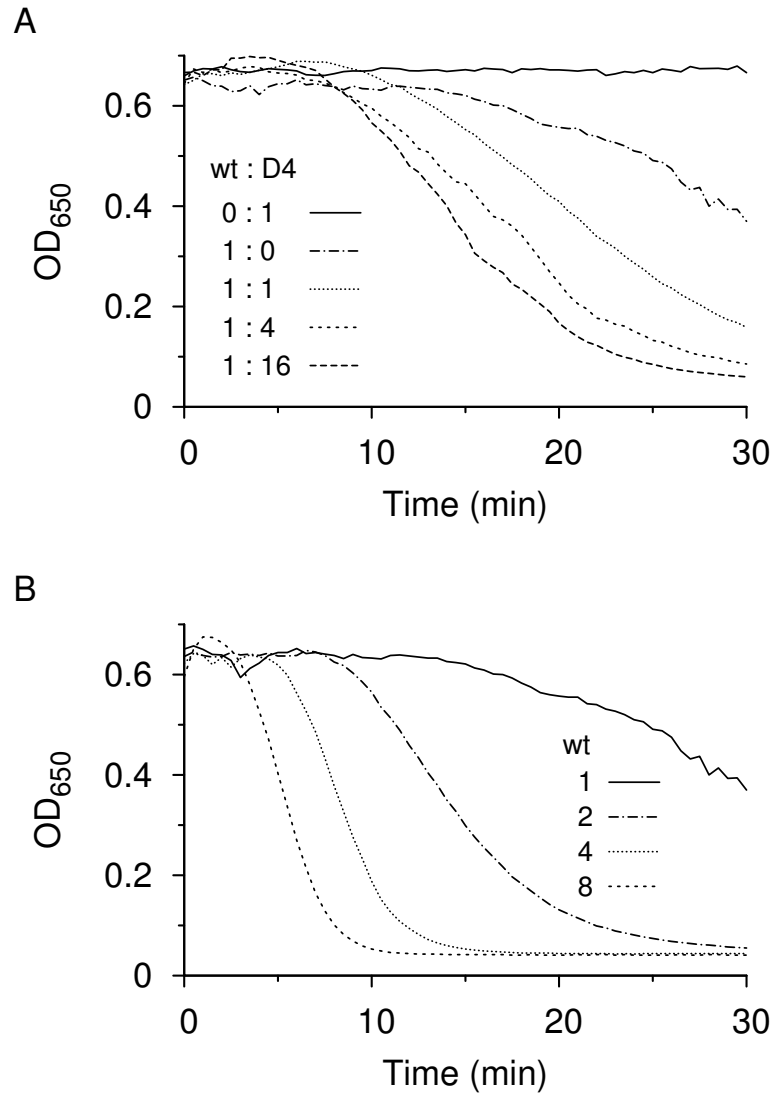
Figure 4

A

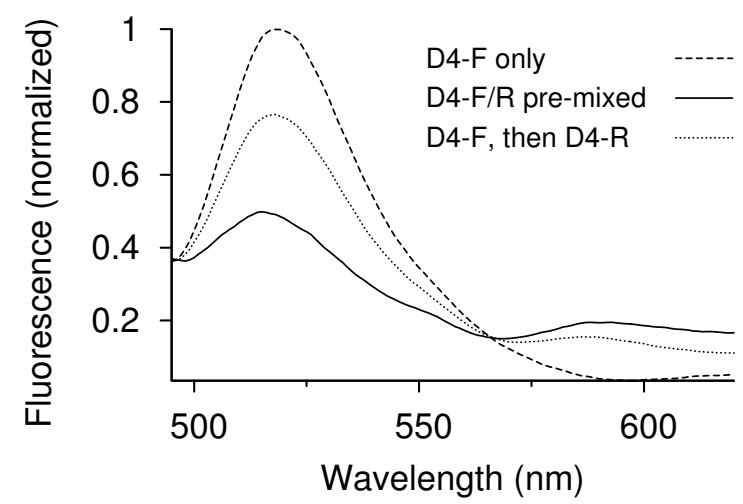

B

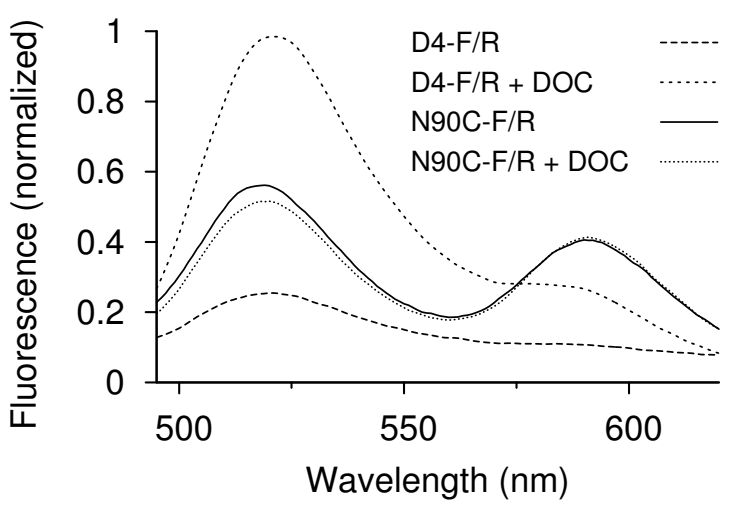


Figure 5
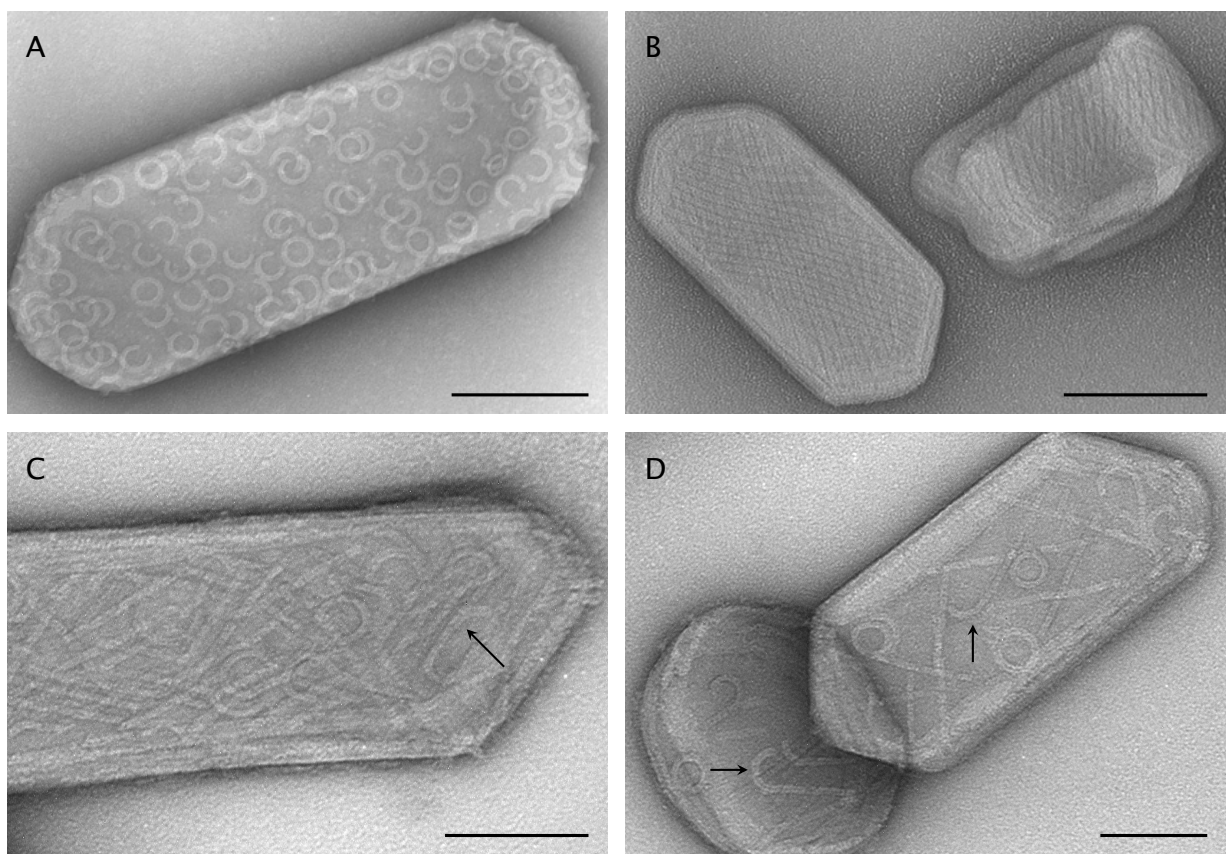
Figure 6

A

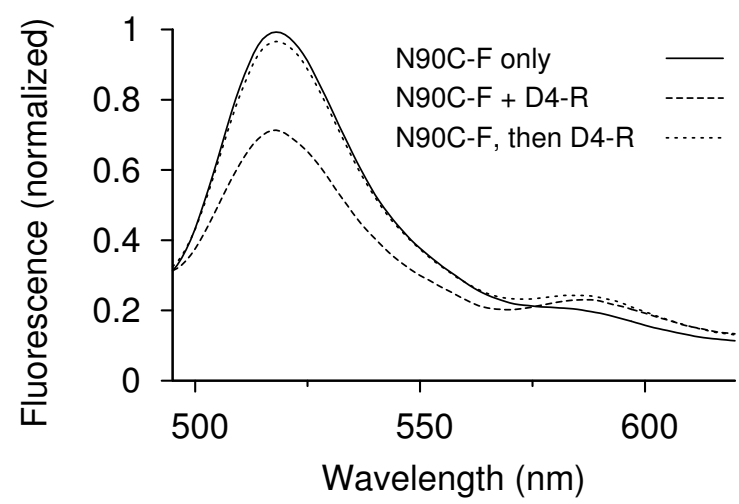

B

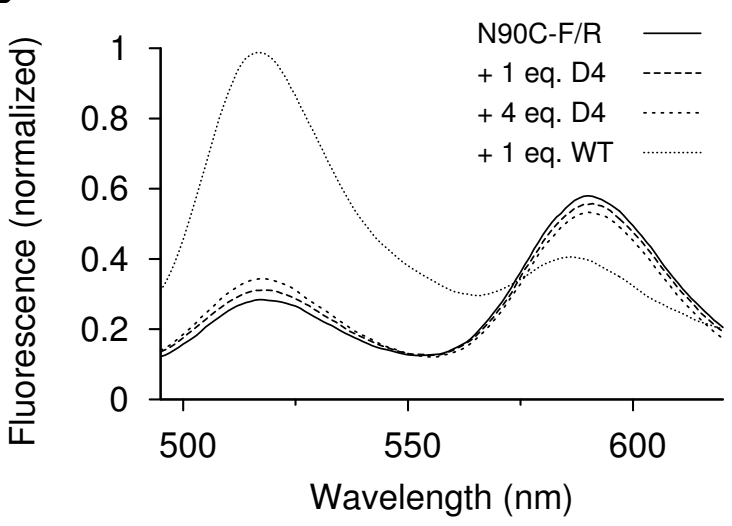


Figure 7

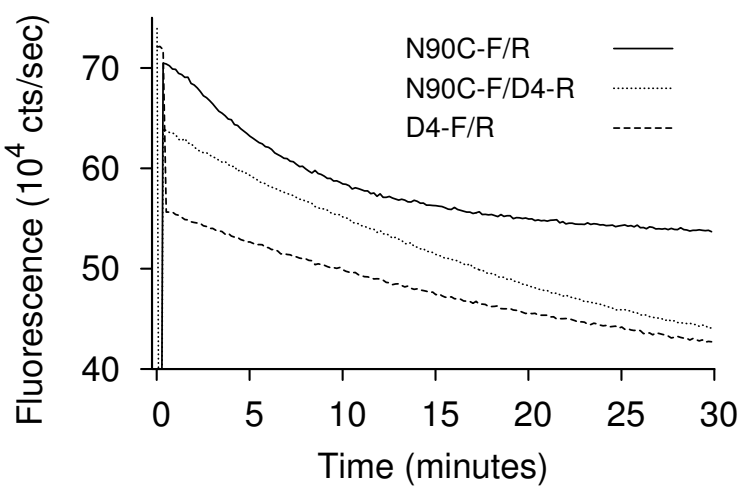


Figure 8

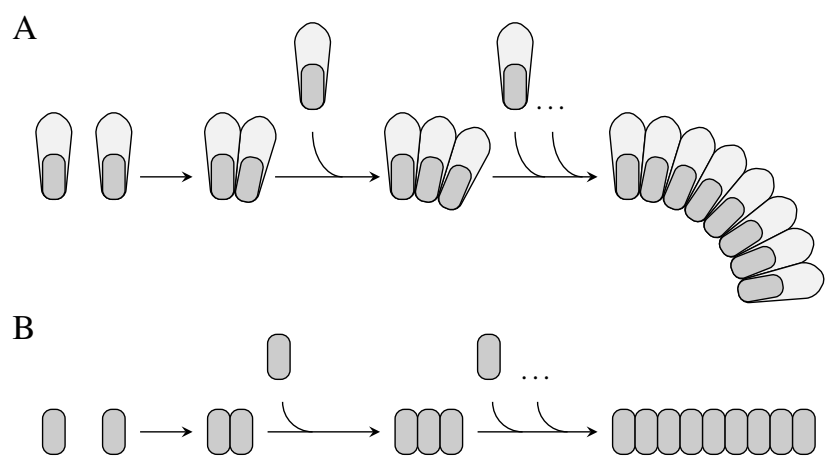

C

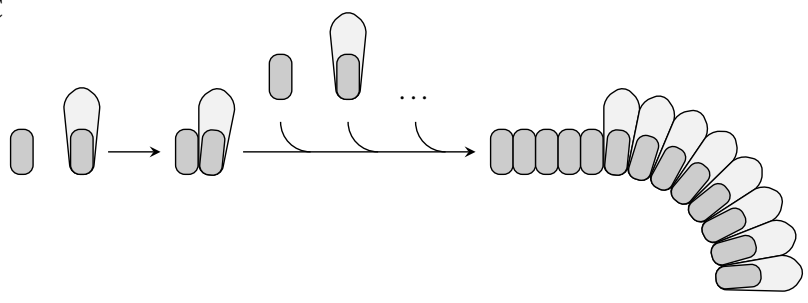

D
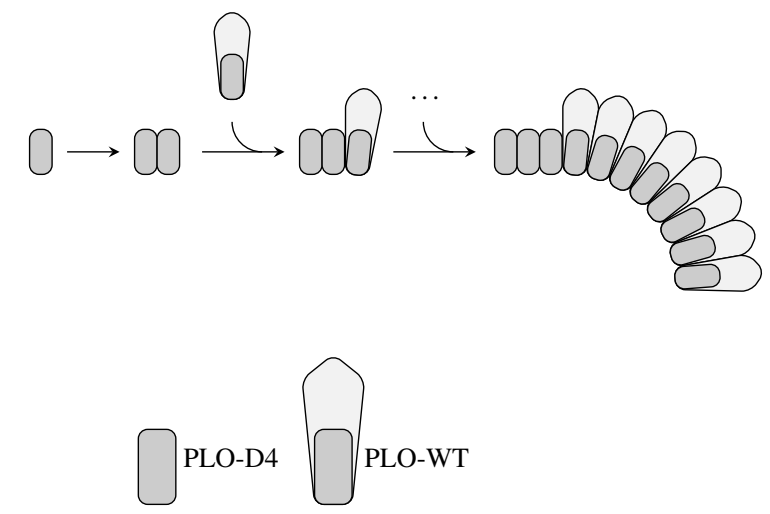